\title{
Determination of Sitagliptin Levels in Rats Serum by HPLC and its Pharmacokinetic Investigation in Existence of Sucralose
}

\author{
Wael Abu Dayyih*, Mohammed Hamad²
}

${ }^{1}$ Dept of Pharmaceutical Medicinal Chemistry and Pharmacognosy, Faculty of Pharmacy and Medical Sciences, University of Petra, Queen Alia International Road, Amman - Jordan 11196, Jordan 2 Dept of Basic Sciences, Collage of Sciences and Health Professions, King Saud Bin Abdulaziz University for Health Sciences, Jeddah 21423, Saudi Arabia

Submitted: $12-06-2018$

Revised: 29-07-2018

Accepted: 11-08-2018

*Corresponding author Wael Abu Dayyih

Email: wabudayyih@uop.edu.jo

\begin{abstract}
It is an interest of the current work to develop a simple, valid and rapid chromatographic method for the quantification of sitagliptin in rats' serum. Accordingly, the pharmacokinetic parameters of sitagliptin was investigated for any possible interaction between sitagliptin and sucralose. The developed HPLC method was validated for sitagliptin determination in rat's serum. Results indicated an accomplished overall intra-day precision and accuracy values of $0.139-4.028$ (CV\%) and 99.48-107.12\% respectively. On the other hand, inter-day precision and accuracy results recorded values of $0.150-2.810$ (CV\%) and 99.90$116.00 \%$ respectively. Moreover, the coefficient of correlation was determined at a value of 0.99950 with reasonable sensitivity and selectivity. Effect of sucralose showed strong significant correlation on sitagliptin serum profile. The precise, accurate, linear, sensitive and selective HPLC method will be elaborated in the context of the current work with emphasis on sucralose negative effect on sitagliptin level in rat's serum. Such finding is a guidance for physicians to reconsider the use of such sweeteners for diabetic patients
\end{abstract}

Key words: sitagliptin, sucralose, tadalafil, pharmacokinetic, interaction.

\section{INTRODUCTION}

Sitagliptin is a dipeptidyl peptidase-4 (DPP-4) inhibitor drug that has been introduced in 2006 for the treatment of type- 2 diabetes. As an inhibitor, its main mechanism of action depends primarily on the suppression of glucagon like peptide-1 (GLP-1) and glucose dependent insolinotropic polypeptide (GIP) actives and thus leading to the increase of insulin secretion (Herma et al., 2006; Abu Dayyih et al., 2015; Dubla et al., 2012; Pathade et al., 2011; Khan et al., 2011).

Sitagliptin, $\mathrm{PO}_{4},(\mathrm{STGPN})$ has a typical gliptin structure (Figure 1) and is chemically known as 1,2,4-triazolo[4,3-a] pyrazine,7-[(3R)3-amino-1-oxo-4-(2,4,5-trifluorophenyl)butyl]5,6,7,8-tetrahydro-3-(trifluoromethyl)-, phosphate (Herma et al., 2006; El-Bagary et al., 2011; Pulla et al., 2011; Shyamala et al., 2011).

On the other hand, non-caloric sugar substitutes have become common food and beverage products that are widely used by type II diabetic patients. Amongst these sucrose derivative compounds, sucralose (SUCS) -a synthetic trichlorinated disaccharide- has been accepted as a safe sweetener by international regulatory bodies. However, sweeteners, in general, are not regarded as biological inert ingredients. Moreover, their pharmacokinetics and drug interaction have not been thoroughly investigated. There are, however, recent studies which proved that sucralose alters physiological parameters, most importantly the ones associated with increased expression of glucagon-like peptide- 1 and with induced CYP3A4 enzyme activity in intestine and liver (Patil et al., 2010; Nikolaou et al., 2011; Zeng et al., 2010; Zeng et al., 2008; Niroji et al., 2008). Such parameters were reported to cause reduction in the bioavailability, pharmacokinetic, and pharmacodynamic activities of certain drugs.

However, the impact of sucralose on the chemical integrity and thus bioavailability of sitagliptin has not been investigated so far. 


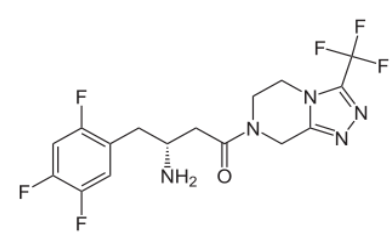

a

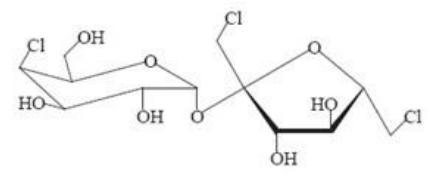

$\mathrm{b}$

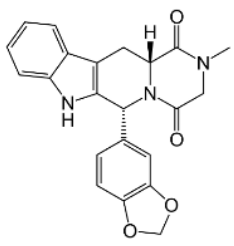

Figure 1. Chemical structures of (a) sitagliptin (STGPN), (b) sucralose (SUCS), (c) tadalafil.

It is suggested that such delay in drug-sweetener studies is attributed to the lack of simple analytical method for the determination of sitagliptin in plasma and serum (El-Bagary et al., 2011; Patil et al., 2010; Vincent et al., 2007; Beconi et al., 2007).

Methods for sitagliptin assay and related compounds determination according to EMEA guidelines were not given a substantial attention when searching for a realistically feasible technique (Beconi et al., 2007; EMA, 2011; Matsubara et al., 2004; Tamimi et al., 2014; Abu Dayyih et al 2018). The foregoing was based on the fact that the only method most commonly discussed on sitagliptin analytical determination in plasma and urine of humans, rats and dogs was a high-performance liquid chromatography with tandem mass spectrometry (LC-MS/MS).

However, although MS gives a highly accurate determination of assay and related values of the drug, its high cost still represents a constraint in equipment availability on a broad range of applications. Further looking into less sophisticated techniques, Ultra-violet (UV) spectroscopic determination of sitagliptin was found to be more appropriately considered for assay determination of the drug. However, in addition to its incapability to test drugs in serum, UV methods cannot distinguish between the drug and its impurities and therefore this method was, and is generally, not considered for related compound determination. Consequently, an alternative technique became a must to develop herein taking a challenge to analytically quantify the drug and its related compounds within the serum (Pathade et al., 2011; Pulla et al., 2011; Zeng et al., 2008; Dayyih et al., 2018).

Many methods had been developed to detect and measure sitagliptin; RP-HPLC Method (Dubal et al., 2012; Shyamala et al., 2011; Patil et al., 2010), Sensitive liquid chromatography tandem mass spectrometry method (Zeng et al.,
2010; Nirogi et al., 2008), GC/MS method (Nikolaou et al., 2011), Spectroflourometric and spectrophotometric methods (El-Bagary et al., 2011) and UV Spectrophotometric Method (Pathade et al., 2011)

In light of the above need for an alternative drug-quantification technique, the current research highlights the application of a new rapid high-performance liquid chromatography-ultraviolet method to monitor any sitagliptin-sucralose interactions. The new method will be investigated according to EMEA guidelines for the quantification of sitagliptin in rats' serum. Tadalafil (TDFL) -a selective inhibitor of cyclic guanosine monophosphate (cGMP)-specific phosphor-diesterase type 5 (PDE5) will be used as an internal standard.

The current research is highlighting on developing of a new simple, sensitive, precis and accurate chromatographic method for measuring sitagliptin in rat's plasma, to be applied to investigate any possible sitagliptinsucralose interactions in rat's plasma.

\section{MATERIAL AND METHODS Chemicals}

HPLC grades of methanol and phosphate buffer were used as the mobile phase solvents for HPLC analysis (FULL-TIME, USA), deionized water HPLC grade was obtained from (TEDYA, USA), STGPN raw material ( $\geq 98 \%)$ was thankfully donated from DAD company (Amman, Jordan). Tadalafil citrate $(\geq 98 \%)$ as the internal standard was purchased from TQ. Pharma. (Amman, Jordan), SUCS was acquired from its finished product Splenda ${ }^{\circledR}($ Tate \& Lyle, Newham, London, UK).

\section{Apparatus and chromatographic}

High performance liquid chromategraphy- ultraviolet HPLC/UV was the technique adopted in this study due to its 
successful performance reported in the analysis of several compounds in plasma or serum (Abu Dayyih et al., 2015; Zeng et al., 2010; Zeng et al., 2008; Nirogi et al., 2008; Vincent et al., 2007; Biconi et al., 2007). The HPLC system (HITACHY, Japan) consisted of S \# L-2130 VWR-HITACHY pump model, S\# L-2200 VWR-HITACHY auto-sampler thermostat, S\# L-2300 VWR-HITACHY column oven and S\# L-2420 VWR-HITACHY- UV detector.

Chromatographic separation was carried out using a mobile phase which consisted of $(65: 35) \mathrm{v} / \mathrm{v}$ phosphate buffer and methanol of a $\mathrm{pH}$ value adjusted at 3.9. The method of HPLC assigned was as follows; the type of separation column used was ACE C18 $(5 \mu \mathrm{m}$, $250 \times 4.6 \mathrm{~mm}$ i.d.) operating at a temperature fixed at $40^{\circ} \mathrm{C}$, an injection volume of $50 \mu \mathrm{L}$, a mobile phase flow rate of $0.001 \mathrm{~L} / \mathrm{min}$, and a sample run time of $9 \mathrm{~min}$. Signals were monitored and analyzed at $\lambda=239 \mathrm{~nm}$. Tadalafil citrate was used as internal standard.

\section{Preparation of stock and working solutions}

Oral STGPN was given at a recommended dose $1.90 \mathrm{~g} / \mathrm{kg} /$ day) for rats. It was prepared by dissolving the drug in methanol. On the other hand, SUCS, given at an oral dose of $(10 \mathrm{mg} / \mathrm{kg} /$ day $)$, was prepared as a working solution of $0.25 \mathrm{~g} / \mathrm{mL}$ by dissolving Splenda ${ }^{\circledR}$ at room temperature under sonication. Both working solutions were injected from fresh preparations that are kept away from heat and light (Abu dayyih et al., 2015; Dubal et al., 2012; Abu Dayyih et al., 2018) (Abu Dayyih et al., 2018; 2015; 2012).

STGPN stock solution was prepared at a concentration of $500 \mu \mathrm{g} / \mathrm{mL}$ by dissolving $25 \mathrm{mg}$ in $50 \mathrm{~mL}$ methanol. STGPN working solutions, preserved from light and heat, were prepared from the stock solution by dilution with methanol then injected directly for HPLC analysis. Tadalafil (TDLF) was used as internal standard.

\section{Calibration samples}

A series of dilutions of samples were prepared by completing an appropriate volume of each previous preparation with a sufficient volume of rat's serum to reach a final volume of $5 \mathrm{~mL}$ at the corresponding calibration concentration. Each sample was vortexed and directly injected for HPLC analysis. Six calibration curves were analyzed using the HPLC. In each calibration curve, peak area ratios were calculated and plotted against the concentrations with a correlation coefficient $\mathrm{R}^{2}$ accepted not to exceed a value of 0.99 .

\section{Sample preparation (extraction procedure)}

Sample preparation was carried out by mixing $100 \mu \mathrm{L}$ of serum with $75.0 \mu \mathrm{L}$ of internal standard (IS) working solution. The sample was then vortexed for $30 \mathrm{~s}$, centrifuged at $12000 \mathrm{rpm}$ for $5 \mathrm{~min}$ and transferred into rack. $50 \mu \mathrm{L}$ of the supernatant was injected into HPLC unit.

\section{Precision and accuracy}

Precision and accuracy of the current method of analysis were evaluated on four different concentrations; lower limit of quantification, low, middle and high concentrations of STGPN by spiking with the rat's serum.

Intra-day precision and accuracy were evaluated by analyzing the four aforementioned concentrations. This was carried out five times in the same day and then repeated at three different days for the inter-day precision and accuracy evaluation. Furthermore, precision was assessed by comparing each concentration with the $\mathrm{CV} \%$ (coefficient of variation) while accuracy was assessed by comparing the mean calculated concentration with $\pm 20 \%$ of spiked concentration for the lower limit of quantification while it was compared with \pm $15 \%$ for the other quality control samples.

\section{Stability}

Different stability conditions were adopted in the current work. For the room temperature stability, three samples of quantification control low concentration (QCL) and quantification control high concentration $(\mathrm{QCH})$ of concentrations 2 and $12.8 \mu \mathrm{g} / \mathrm{mL}$ respectively, were prepared and analyzed with freshly prepared calibration at zero time and after $8 \mathrm{~h}$ at room temperature of $28^{\circ} \mathrm{C}$. Percent area ratios per QCL and QCH were calculated and each corresponding calibration was constructed (Abu Dayyih et al., 2015; Khan et al., 2011; Beconi et al., 2007; Dayyih et al., 2018). 
For freeze and thaw stability, three samples of QCL and QCH were properly prepared in serum with sufficient final volume covering all test cycles of analysis. For the zerotime stability, for each corresponding calibration, samples were analyzed, and concentrations were calculated, then, samples were stored and frozen at $-20^{\circ} \mathrm{C}$. For the $12 \mathrm{~h}$ stability, samples were thawed at room temperature, i.e. $25^{\circ} \mathrm{C}$, corresponding to the samples processing temperature during analysis stages. After complete thawing, samples were analyzed again for each corresponding calibration, then refrozen for $24 \mathrm{~h}$. For the $24 \mathrm{~h}$ stability, samples were thawed and analyzed under the same conditions. After each cycle, concentrations were calculated relative to its corresponding calibration. For the longterm stability, three samples of (QCL and QCH) were analyzed with freshly prepared calibration at zero time. Samples were then frozen for 30 days at a temperature of $-20^{\circ} \mathrm{C}$. After the long term freezing period was complete, samples were removed and thawed at room temperature.

Room temperature and long-term stability test were also carried out for the working solutions. Stability test was assessed by comparing the calculated concentrations with the nominated (or assigned) concentrations as it should be within $\pm 15 \%$ for both QCL and QCH.

\section{Preclinical study}

The preclinical study protocol was approved by the Research Committee (October 14/6/2016) at the Faculty of Pharmacy and Medical Sciences, University of Petra, Amman, Jordan.

Adult male Sprague Dawley laboratory rats were supplied by Petra University Animal House. Average weight of rats was $(0.230 \mathrm{~kg} \pm$ $0.03)$. Rats were placed in air-conditioned environment at a temperature of $20-25^{\circ} \mathrm{C}$ and exposed to a photoperiod cycle (12h light /12-h dark) at a humidity of $50 \%$ on daily basis. Rats were under fasting for $24 \mathrm{~h}$ and weighed directly before the experiment. All used rats were in healthy conditions before and after experiment as rats were monitored for one-month post analysis. STGPN and SUCS test solutions were freshly prepared directly in the laboratory before rats feeding in order to avoid any possible decomposition of either STGPN or SUCS.

A total of 80 healthy rats were used for the experiment. Rats were divided into groups of 8 rats, then weighed and numbered orderly. STGPN and SUCS oral doses were calculated based upon each rat's weight then administered according to their numbers in order using gastric gavage. Trial analysis was performed in 3 days according to the following arrangement:

In the first day of trials: Four groups of rats were used for analysis; two groups received water at zero-time experiment, then followed by STGPN after $1 \mathrm{~h}$ of water feeding, while the other two group received SUCS at zero time of experiment followed by STGPN after $1 \mathrm{~h}$ of SUCS feeding. Time intervals of blood samples pooling were: $0,30 \mathrm{~min}, 1 \mathrm{~h}, 2 \mathrm{~h}, 3 \mathrm{~h}, 4 \mathrm{~h}, 6 \mathrm{~h}, 8 \mathrm{~h}$ and $24 \mathrm{~h}$.

At second day of trials: Two groups received water at zero time of experiment followed by STGPN after one hour, while other two groups received SUCS at zero time followed by STGPN after $1 \mathrm{~h}$ of SUCS feeding. Time intervals of blood samples pooling were: 0 , $30 \mathrm{~min}, 1 \mathrm{~h}, 2 \mathrm{~h}, 3 \mathrm{~h}, 4 \mathrm{~h}, 6 \mathrm{~h}, 8 \mathrm{~h}$ and $24 \mathrm{~h}$.

At third day of trials: Two groups received water at zero time of experiment followed by STGPN after one hour, while the other two groups received SUCS at zero time followed by STGPN after $1 \mathrm{~h}$ of SUCS feeding. Time intervals of blood samples pooling were: 0 , $30 \mathrm{~min}, 1 \mathrm{~h}, 2 \mathrm{~h}, 3 \mathrm{~h}, 4 \mathrm{~h}, 6 \mathrm{~h}, 8 \mathrm{~h}$ and $24 \mathrm{~h}$.

Tail tip of each rat was cut after weighing and numbering, approximately $200 \mu \mathrm{L}$ of blood was pooled into Eppendorf tube at each time interval under the same numbering order, after total time intervals of blood pooling was finished, samples were centrifuged for $10 \mathrm{~min}$ (12000rpm) to obtain pure serum that was needed for analysis then frozen at $-20^{\circ} \mathrm{C}$.

\section{RESULTS AND DISCUSSIONS}

Separation between chromatogram peaks between STGPN and Tadalafil in serum was satisfactorily attained. Individual peaks of STGPN were sharp, clear of tailing or splitting, of reasonable retention time and high resolution. An overlay chromatogram of all the aforementioned peaks in samples representing blank rat's serum, rats serum with IS, rats serum 

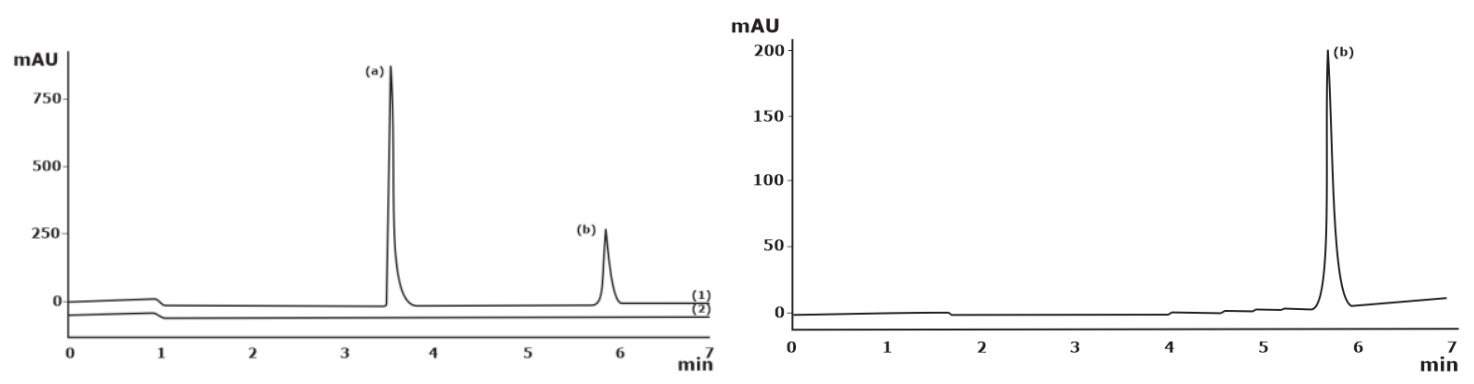

Figure 2. (A) Blank chromatogram (1) and separation chromatogram (2): STGPN (peak b) and internal standard Tadalafil citrate (peak a) chromatogram (B) STGPN chromatogram.

Table I. Linearity of six calibration curves.

\begin{tabular}{ccc}
\hline Calibration no. & Calibration equation & Correlation coefficient $\left(\mathbf{R}^{\mathbf{2}}\right)$ \\
\hline Calibration 1 & $\mathrm{y}=0.1008981 \mathrm{x}-0.00098912$ & 0.999890 \\
Calibration 2 & $\mathrm{y}=0.1092190 \mathrm{x}+0.00043396$ & 0.999777 \\
Calibration 3 & $\mathrm{y}=0.0995990 \mathrm{x}-0.001155060$ & 0.999168 \\
Calibration 4 & $\mathrm{y}=0.1013354 \mathrm{x}-0.005413047$ & 0.999991 \\
Calibration 5 & $\mathrm{y}=0.0987726 \mathrm{x}+0.00257729$ & 0.998992 \\
Calibration 6 & $\mathrm{y}=0.0966405 \mathrm{x}-0.01064450$ & 0.999113 \\
\hline
\end{tabular}

Table II. Inter- day precision and accuracy over three days, and intra-day precision and accuracy for QC samples.

\begin{tabular}{cccccc}
\hline \multicolumn{7}{c}{ Inter-day: Day 1 } \\
\hline Sample ID & Mean & SD & CV\% & Error & Accuracy \% \\
\hline ST(LLOQ) & 0.264 & 0.0046 & 1.782 & 0.011 & 104.011 \\
QCL & 1.998 & 0.0038 & 0.193 & 0.013 & 99.961 \\
QCM & 7.103 & 0.0915 & 1.301 & 0.104 & 101.431 \\
QCH & 13.51 & 0.1229 & 0.910 & 0.15 & 105.541 \\
\hline \multicolumn{5}{c}{ Inter-day: Day $\mathbf{~}$} \\
\hline ST(LLOQ) & 0.254 & 0.0042 & 1.45 & 0.011 & 100.10 \\
QCL & 2.017 & 0.0031 & 0.16 & 0.020 & 100.01 \\
QCM & 7.314 & 0.0980 & 1.35 & 0.311 & 104.2 \\
QCH & 13.522 & 0.4503 & 3.34 & 0.601 & 105.1 \\
\hline \multicolumn{5}{c}{ Inter-day: Day 3 } \\
\hline ST(LLOQ) & 0.256 & 0.0039 & 1.56 & -0.0039 & 100.03 \\
QCL & 2.144 & 0.0090 & 0.43 & 0.04321 & 107.0 \\
QCM & 7.328 & 0.0857 & 1.18 & 0.4222 & 104.58 \\
QCH & 13.715 & 0.3853 & 2.82 & 1.0116 & 107.12 \\
\hline \multicolumn{5}{c}{ Intra-day } \\
\hline ST(LLOQ) & 0.260 & 0.0036 & 1.36 & 0.011 & 104.02 \\
QCL & 1.991 & 0.0028 & 0.15 & -0.012 & 102.21 \\
QCM & 7.164 & 0.0960 & 1.35 & 0.164 & 103.94 \\
QCH & 13.312 & 0.5364 & 4.04 & 0.516 & \\
\hline
\end{tabular}


with Low LOQ, and rat's serum with IS and STGPN after $30 \mathrm{~min}$ of combination, respectively (Figures 2).

Method performance attributes including precision, accuracy, and linearity were carried out and statistically tested over six replications. Calibration curves were constructed over six different trials (Table I) resulting in a correlation coefficient greater than 0.999 thus confirming the good linearity that was established between the peak area of STGPN in serum and its concentrations. Precision of inter- and intra- day tests demonstrated acceptable lower limit of quantification (LLOQ) values with coefficient of variation $(\mathrm{CV} \%)$ determined to be less than $20 \%$. For the quantification control concentrations representing low (QCL), medium (QCM) and high (QCL) values for the six runs, a coefficient of variation less than 15\% was recorded (Table II). On the other hand, inter-day and intra-day tests showed accuracy values with lower limit of quantification (LLOQ) lying within a CV\% of $\pm 20 \%$ for defined sample concentrations, whilst within $\pm 15 \%$ for the QCL, QCM and QCH`.

Three different conditions were adopted for testing drug stability; Freeze and thaw stability, Room temperature, and Long-term freezing. Stability data of the freeze-dried, samples were obtained when the samples representing QCL and $\mathrm{QCH}$ concentrations were analyzed at zero time, after 12 and $24 \mathrm{~h}$ of freezing. All samples were thawed for $3 \mathrm{~h}$ then analyzed. Assay data indicated $99 \%$ of the drug with accuracy values exceeding 98\% (Figure 3, Table III).

For stability studies at room temperature, serum samples representing QCL and QCH concentrations analyzed at zero time and after $12 \mathrm{~h}$ at room temperature $\left(28^{\circ} \mathrm{C} \pm 1\right)$ had stability and accuracy values exceeding 99\% (Table IV). Finally, for the long term freezing condition, serum samples representing QCL and QCH concentrations analyzed at zero time and after 30 days and thawing for $3 \mathrm{~h}$ had stability and accuracy values exceeding $99 \%$ and $98 \%$ respectively (Figure 3, Table IV).

\section{SUCS -STGPN combination effect on STGPN serum levels:}

Assay of STGPN samples in rat's serum was determined at each day of trials. Samples representing the first day of trials were collected at zero time and at $24 \mathrm{~h}$. Assay results (Figure 4A), while samples representing the last time interval (Table V). Samples collected on the second day of trials (Figure 4B) were analyzed at zero time and $24 \mathrm{~h}$ (Table V). Samples collected on the third day of trials had an overall serum concentration profiles represented by area under the curve (AUC), maximum concentration (C-max), and maximum time (T-max) (Table $\mathrm{VI})$.

Statistical treatment of the samples was carried out to evaluate size and significance in the combination of STGPN and SUCS in the oral dose (is this correct). Effect size was expressed by Cohen's d value while the $P$ value was used to indicate significance. Cohen's d values for the combination effect size did not exceed 0.8 for all intervals except at $30 \mathrm{~min}$ where lower $\mathrm{d}$ values were recorded. With regard to $P$ values, combination effect significance was strong as all $P$ values were less than 0.01 . Finally, two examples on the designed procedure for HPLC analysis are illustrated in the chromatograms (Figure 3 and 4).

Method validation was evaluated for quantitative purposes according to EMEA guidelines (Herma et al., 2006; Dayyih et al., 2018). Concerning precision and accuracy, values determined were reasonably acceptable as indicated by the low $\mathrm{CV} \%$ values obtained. In addition, Method linearity established an accepted correlation coefficient confirmed above 0.999 .

Accordingly, the three aforementioned stability tests verified the reliability of the HPLC procedure to analyze the drug with or without the presence of SUCS with reasonable accuracy and precision data obtained. In consequence, such a validated method gives more emphasis to the use of the current method as a replacement to most widely used HPLC procedures which are highly expensive and more complex to operate.

Having a SUCS -STGPN combination being investigated for the first time in the current research, the initial statistical analysis of the data presented herein suggests a significant interaction between the two compounds when administered concurrently. Such interaction was supported by a $P$ value test outcome $<0.01$ for the combination effect which indicates 
Wael Abu Dayyih

Table III. Freeze and thaw stability for QCL and QCH.

\begin{tabular}{|c|c|c|c|c|c|c|}
\hline \multicolumn{4}{|c|}{ QC Low $(2 \mu \mathrm{g} / \mathrm{mL})$} & \multicolumn{3}{|c|}{ QC High $(12.8 \mu \mathrm{g} / \mathrm{mL})$} \\
\hline Time & Mean & Accuracy $\%$ & Stability $\%$ & Mean & Accuracy $\%$ & Stability \% \\
\hline \multirow{4}{*}{$\mathrm{Oh}$} & & 103.10 & & & 99.69 & 99.30 \\
\hline & $2.012 \pm 0.112$ & 102.01 & 100.60 & $12.711 \pm 2.322$ & 99.90 & \\
\hline & & 99.84 & & & 99.80 & \\
\hline & & 99.98 & & & 98.91 & \\
\hline \multirow[t]{3}{*}{$12 \mathrm{~h}$} & $1.981 \pm 0.210$ & 99.98 & 98.46 & $12.753 \pm 3.112$ & 98.80 & 100.33 \\
\hline & & 99.99 & & & 99.00 & \\
\hline & & 99.97 & & & 98.70 & 99.85 \\
\hline \multirow[t]{2}{*}{$24 \mathrm{~h}$} & $1.990 \pm 0.096$ & 99.99 & 99.50 & $12.692 \pm 1.996$ & 98.88 & \\
\hline & & 99.89 & & & 98.90 & \\
\hline
\end{tabular}

Table IV. Room temperature and Long-term stability of STGPN in serum for QCL and QCH.

\begin{tabular}{|c|c|c|c|c|c|c|}
\hline \multicolumn{7}{|c|}{ Room temperature } \\
\hline \multicolumn{4}{|c|}{ QC Low $(2 \mu \mathrm{g} / \mathrm{mL})$} & \multicolumn{3}{|c|}{ QC High $(12.8 \mu \mathrm{g} / \mathrm{mL})$} \\
\hline Time & Mean & Accuracy $\%$ & Stability $\%$ & Mean & Accuracy $\%$ & Stability $\%$ \\
\hline \multirow{3}{*}{ Oh } & & 110.1 & & & 99.91 & \multirow{3}{*}{100.15} \\
\hline & $2.11 \pm 0.068$ & 106.1 & 105.50 & $12.82 \pm 3.126$ & 100.12 & \\
\hline & & 100.2 & & & 99.91 & \\
\hline \multirow{3}{*}{$8 \mathrm{~h}$} & \multirow{3}{*}{$2.29 \pm 1.001$} & 105.3 & & & 99.84 & \multirow{3}{*}{99.69} \\
\hline & & 99.5 & 108.53 & $12.78 \pm 1.735$ & 99.82 & \\
\hline & & 99.6 & & & 99.77 & \\
\hline \multicolumn{7}{|c|}{ Long-term stability } \\
\hline \multicolumn{4}{|c|}{ QC Low $(2 \mu \mathrm{g} / \mathrm{mL})$} & \multicolumn{3}{|c|}{ QC Low $(2 \mu \mathrm{g} / \mathrm{mL})$} \\
\hline Time & Mean & Accuracy $\%$ & Stability $\%$ & Mean & Accuracy $\%$ & Stability $\%$ \\
\hline \multirow{3}{*}{ Oh } & \multirow{3}{*}{$1.98 \pm 1.002$} & 99.72 & \multirow{3}{*}{99.00} & \multirow{3}{*}{$12.76 \pm 1.886$} & 98.89 & \multirow{6}{*}{99.88} \\
\hline & & 99.25 & & & 98.51 & \\
\hline & & 99.74 & & & 99.35 & \\
\hline \multirow{3}{*}{ 30days } & & 100.90 & & & 99.95 & \\
\hline & $1.99 \pm 0.196$ & 99.91 & 100.50 & $12.68 \pm 2.093$ & 99.27 & \\
\hline & & 99.50 & & & 98.81 & \\
\hline
\end{tabular}

Table V. Three days data for both drug alone and drug with SUCS ( $n=2)$.

\begin{tabular}{lccccccccc}
\hline & \multicolumn{7}{c}{ Sirst day data } \\
\cline { 2 - 9 } & \multicolumn{7}{c}{ Serum average concentrations $(\boldsymbol{\mu g} / \mathbf{m L})$} \\
\cline { 2 - 9 } & $\mathbf{3 0 \mathbf { m i n }}$ & $\mathbf{1 h}$ & $\mathbf{2 h}$ & $\mathbf{3 h}$ & $\mathbf{4 h}$ & $\mathbf{6 h}$ & $\mathbf{8 h}$ & $\mathbf{2 4} \mathbf{~ h}$ \\
\hline Group 1+2 (STGPN alone) & 5.515 & 10.991 & 15.140 & 13.004 & 10.940 & 8.942 & 6.204 & 0.512 \\
Group 3+4 (STGPN+ SUCS) & 5.007 & 10.017 & 12.081 & 11.088 & 10.030 & 7.013 & 4.650 & 0.201 \\
& & \multicolumn{7}{c}{ Second day data } \\
Group 1+2 (STGPN alone) & 5.982 & 10.687 & 15.299 & 13.144 & 12.492 & 9.628 & 6.899 & 0.580 \\
Group 3+4 (STGPN+ SUCS) & 5.147 & 10.275 & 12.131 & 11.242 & 10.126 & 8.036 & 5.146 & 0.314 \\
& & \multicolumn{7}{c}{ Third day data } \\
Group 1+2 (STGPN alone) & 5.893 & 10.671 & 15.982 & 13.257 & 12.097 & 10.802 & 6.691 & 0.596 \\
Group 3+4 (STGPN+ SUCS) & 5.683 & 10.401 & 12.024 & 11.026 & 10.714 & 8.708 & 5.012 & 0.303 \\
\hline
\end{tabular}




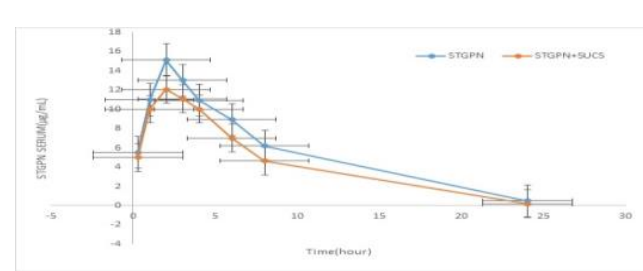

a

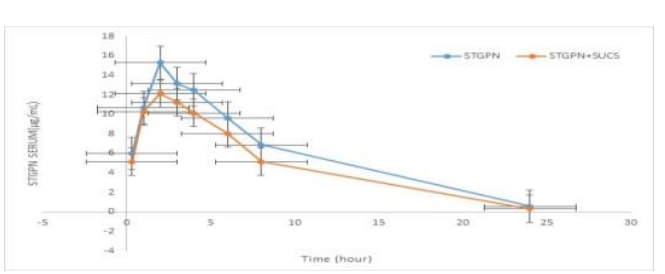

b

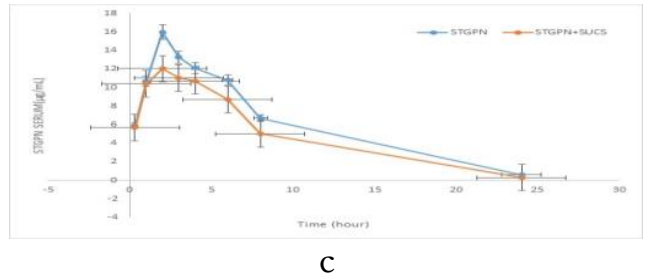

Figure 3. a Freeze and thaw stability chromatogram b Long-term stability chromatogram.
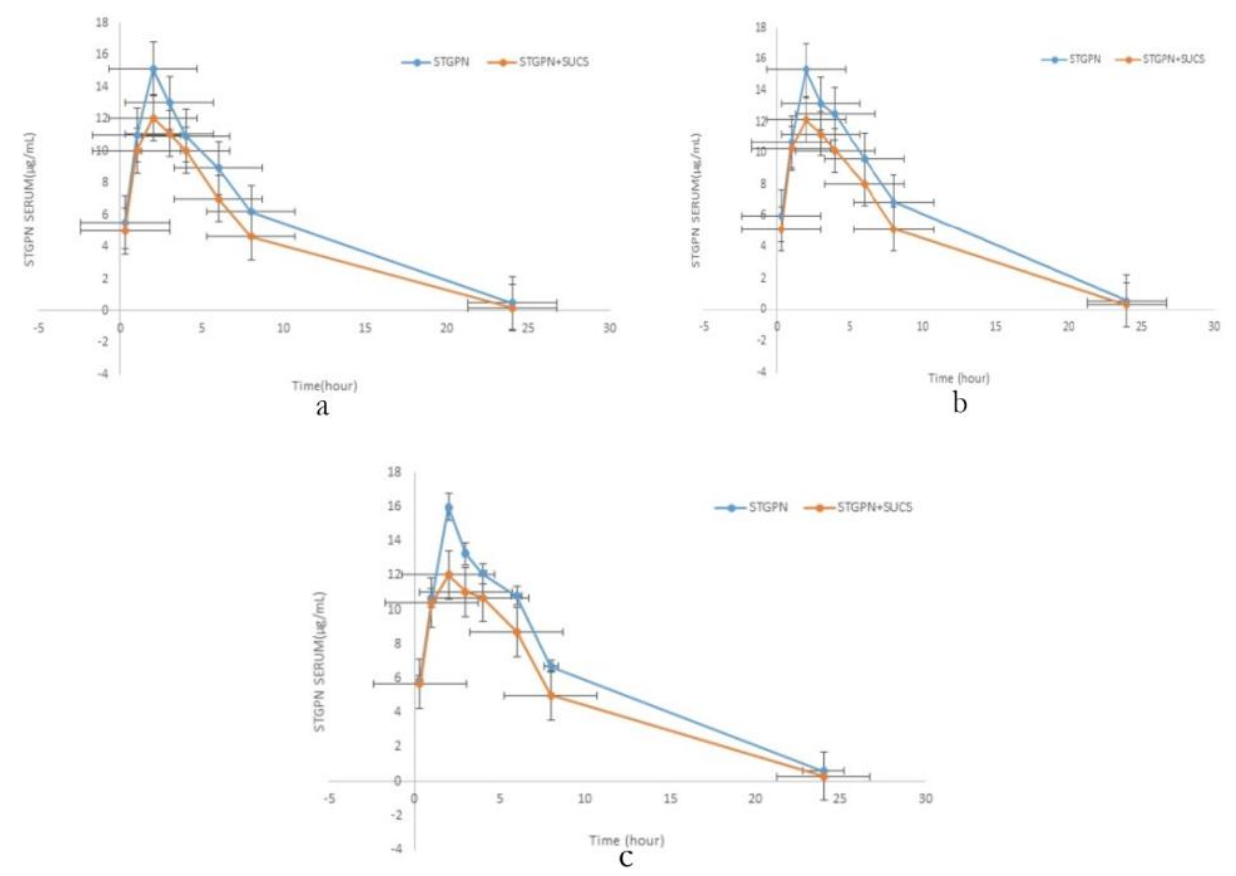

Figure 4 a. First day; b. Second day; c. Third day trial serum-time profile curves.

Table VI. Serum concentration - time profile kinetic parameters.

\begin{tabular}{|c|c|c|c|c|}
\hline Drug & $\mathrm{C} \max (\mu \mathrm{g} / \mathrm{mL})$ & $T \max (h)$ & T $1 / 2(h)$ & $\operatorname{AUC}\left(\mu \mathrm{g} / \mathrm{mL}^{*} \mathrm{~h}\right)$ \\
\hline STGPN & $15.238 \pm 0.029$ & 2 & 2 & $130.7 \pm 2.11$ \\
\hline STGPN + SUCS & $12.079 \pm 0.099$ & 2 & 2 & $101 \pm 2.02$ \\
\hline$P$ value & $0.009 * *$ & \multicolumn{2}{|c|}{$<0.01 * *$} & $0.001 * *$ \\
\hline
\end{tabular}

** $\mathrm{p}<0.01$ significance 
a significant interaction to dominate the drop of drug level in serum and increase in drug metabolites.

Referring back to the mechanism of action of SUCS, it is thought that this combination interaction is more likely attributed to CYP 450 enzyme inhibition by the synthetic sweetener, specifically inhibition of the 3A4 subtype by which STGPN is extensively metabolized. Once STGPN is absorbed in the stomach, CYP3A4 iso-enzymes, located profusely in parietal cells endoplasmic reticulum, will induce metabolic biotransformation of STGPN. Moreover, induction effect of SUCS over the CYP3A4 metabolic enzyme will also activate the metabolism of STGPN in the liver. All of the aforementioned inhibitions of enzyme activities will accordingly result in STGPN plasma/serum levels reduction and over production of STGPN active and inactive metabolites.

The CYP3A forms in rats, i.e. the CYP3A1, 3A2, 3A9, 3A18, 3A23 and 3A62 have the CYP3A23 being identical to the CYP3A1 by the analysis of its gene. The CYP3A62 form has been identified as an iso-enzyme with expression profile similar to the human CYP3A4 and rat CYP3A9. In connecting the dots, the CYP3A62 is a known to be the predominant form in the intestinal tract, whereas the CYP3A1 and -3A2 are found only in liver (Matsubara et al., 2004; Tamimi et al., 2014; Dayyih et al., 2018).

Moreover, a recent study has illustrated the presence of a distinctive binding affinity of STGPN to reactive sites of the CYP3A enzymes during metabolic reactions (Vincent et al., 2007; Matsubara et al., 2004; Dayyih et al., 2018). This may further confirm a predicted variable bioavailability in humans as justified by the current observed in-vivo reduction of STGPN in rats. Such finding may lead to a possible perceptible clinical difference in STGPN human plasma levels when combined with SUCS, which strongly recommends further clinical research in humans.

\section{CONCLUSIONS}

A successful HPLC method was validated and developed to quantify STGPN in rats' serum. The method was precise and accurate with rational linearity performance and reasonable sensitivity and selectivity.
Concerning stability and recovery tests, all obtained results were reasonable and accepted according to EMEA guidelines. Combination effect of STGPN with SUCS over all time intervals of STGPN serum profile demonstrated as strong effect on STGPN pharmacikinetic according to Cohen's $\mathrm{d}$ and significant $\mathrm{P}$ values. C-max showed a significant change between presence and absence of SUCS while T-max did not show any change. This suggests the possibility of interaction between STGPN and SUCS during combination.

\section{ACKNOWLEDGMENT}

The author would like to extend their sincere appreciation to Faculty of Pharmacy and Medical Sciences at University of Petra -Jordan

\section{REFERENCES}

Dayyih WA., Tamimi L., Mallah E., Mansour K., Arafat T., Bustami M., 2015. Saxagliptin Levels and its Pharmacokinetic Application in Presence of Sucralose in Animals Serum by HPLC Method. Int. J. Parm. Pharmac. Sci. 7(9): 243-250.

Dayyih WA., Hamad M., Mallah E., Abu Dayyih A., Awad R., et al., 2018. METHOD Development and Validation of Vildagliptin and Metformin $\mathrm{HCl}$ in Pharmaceutical Dosage form by Reversed Phase-High Performance Liquid Chromatography (RP-HPLC), IJPSR. 9(7): 2965-2972.

Beconi MG., Reed JR., Teffera Y., Xia YQ., Kochansky CJ., et al., 2007. Disposition of the dipeptidyl peptidase 4 inhibitor STGPN in rats and dogs. Drug. Metabol. Dispos. 35(4): 525-532.

Dubal A., Khatwal R., Kosaraju J., Meda V., Samanta M., 2012. Bioanalytical method development and validation of STGPN phosphate by RP-HPLC and its application to pharmacokinetic study. Int. J. Parm. Pharmac. Sci. 4(2): 691-694.

El-Bagary RI., Elkady EF., Ayoub BM., 2011. Spectroflourometric and spectrophotometric methods for the determination of STGPN in binary mixture with metformin and ternary mixture with metformin and STGPN alkaline degradation product. Int. J. Biomed. Sci. 7(1): 62-69. 
EMA. Guideline on Bioanalytical Method Validation. EMA, Committee for Medicinal Products for Human Use (CHMP), London, UK (2011).

Herma GA., Bergman A., Liu F., Stevens C., Wang AQ., et al., 2006. Pharmacokinetics and pharmacodynamic effects of the oral DPP-4 inhibitor STGPN in middle-aged obese subjects. J. Clinic. Pharmacol. 46(8): 876-886.

Khan AYP., Sabarwal NJA., Gupta A.K., 2011. Simultaneous Estimation of Metformin and Sitagliptin in tablet dosage form. Asian J Biochem pharmac Res.1(2): 352-358.

Matsubara T., Kim HK., Miyata M., Shimada M., Nagata K., Yamazoe Y., 2004. Isolation and characterization of a new major intestinal CYP3A form, CYP3A62, in the rat. J. Pharmacol. Exper. Therap. 309(3): 1282-1290.

Nikolaou P., Papoutsis I., Athanaselis S., Alevisopoulos G., Khraiwesh A., et al., 2011. Development and validation of a GC/MS method for the determination of tadalafil in whole blood. J. Pharmaceu. Biomed. Analy. 56(3): 577-581.

Nirogi R., Kandikere V., Mudigonda K., Komarneni P., Aleti R., 2008. Boggavarapu, R. Sensitive liquid chromatography tandem mass spectrometry method for the quantification of sitagliptin, a DPP-4 inhibitor, in human plasma using liquidliquid extraction. Biomed. Chromatog. 22(2): 214-222.

Pathade A., Imran M., Bairagi V., Ahire Y., 2011. Development and Validation of Stability Indicating UV Spectrophotometric Method for the Estimation of Sitagliptin Phosphate in Bulk and Tablet Dosage Form. J. Pharmy. Rese. 4(3): 871-873.
Patil S., Ramesh B., Patil K., 2010. Dhokane, A. Development and Validation of RPHPLC Method for the Estimation of STGPN Phosphate in Tablet Dosage Form. Asian. J. Rese. Chem. 3(3): 653-655.

Pulla RP., Sastry BS., Rajendra PY., Appala RN., 2011. Simultaneous Estimation of Metformin $\mathrm{HCl}$ and STGPN Phosphate in Tablet Dosage Forms by RP-HPLC. Res. J. Pharm. Techn. 4(4): 646-649.

Shyamala M., Mohideen S., Satyanarayana T., Narasimha R., Suresh K., Swetha K., 2011. Validated RP- HPLC for simultaneous estimation of Sitagliptin phosphate and Metformin in hydrochloride in tablet dosage form. Ame. J. Pharm.Tech. research. 1(2): 93-101.

Tamimi L., Abu Dayyih W., Qinna N., Mallah E., Arafat T., 2014. Pioglitazone $\mathrm{HCl}$ Levels and Its Pharmacokinetic Application in Presence of Sucralose in Animals Serum by HPLC Method. Pharmace. Analyt. Acta. 5: 318-325.

Vincent SH., Reed JR., Bergman AJ., Elmore CS., Shiyoa Xu EB., et al., 2007. Metabolism and excretion of the dipeptidyl peptidase 4 inhibitor [14C] STGPN in humans. Drug. Metabol. Dispos. 35(4): 533-538.

Zeng W., Xu Y., Constanzer M., Woolf EJ., 2010. Determination of Sitagliptin in human plasma using protein precipitation and tandem mass spectrometry. $J$. Chromatog. B. 878(21): 1817-1823.

Zeng W., Musson DG., Fisher AL., Chen L., Schwartz MS., et al., 2008. Determination of Sitagliptin in human urine and hemodialysate using turbulent flow online extraction and tandem mass spectrometry. J. Pharmaceu. Biomed. Analy. 46(3): 534-542. 